PHYSICAL REVIEW FLUIDS 2, 090507 (2017)

\title{
Fall and fragmentation of liquid metal in a viscous fluid
}

\author{
Jean-Baptiste Wacheul* and Michael Le Bars \\ CNRS, Aix Marseille Univ, Centrale Marseille, IRPHE, 13013 Marseille, France
}

(Received 26 July 2017; published 29 September 2017)

\begin{abstract}
This paper is associated with a video winner of a 2016 APS/DFD Gallery of Fluid Motion Award. The original video is available from the Gallery of Fluid Motion, https://doi.org/10.1103/APS.DFD.2016.GFM.V0038
\end{abstract}

DOI: 10.1103/PhysRevFluids.2.090507

Simulations of the solar system formation and the diversity of meteorite chemical composition both point toward a scenario where the late stages of Earth-like planet accretion involved massive impacts of Mars-size planets [1]. Due to the combination of the energy release during impact, the intense radioactive decay of short-lived elements, and the heat remaining from the conversion of potential energy required to form a planet, these planetary embryos were most likely molten for a large part [2]. This also means that they were differentiated in a liquid iron core covered by a molten silicate mantle, since these two main phases of terrestrial bodies are immiscible and of significantly different densities. Following each impact, a large-scale two-phase flow occurred as the molten iron of the impactor core flowed across the silicate magma ocean to merge with the planetary embryo core. Because of the immense inertia of the process, this flow was characterized by very high Reynolds and Weber numbers and hence prone to rapid fragmentation. The quantification of the exchanges of heat and elements in this dispersed flow is crucial to the knowledge of the initial state of telluric planets.

While the dynamics of rising bubbles have been largely investigated [3], studies of the planetary regime of two-phase flow and fragmentation are sparse. Models of Earth's formation mostly rely on asymptotic models where the initial volume of iron either is immediately spread into an ensemble of drops of capillary sizes that behave independently [4] or remains as a whole until reaching the bottom of the magma ocean, developing Rayleigh-Taylor- and Kelvin-Helmholtz-type instabilities on its sides [5]. Recently, Landeau and co-workers [6,7] performed laboratory experiments showing that in the relevant large-Weber-number limit, the released buoyant fluid behaves initially like a turbulent thermal whose surface of contact with the ambient fluid has a fractal aspect and therefore a large surface area, well before fragmentation and formation of a cloud of droplets.

The viscosity of the magma ocean is a key variable in the geophysical application. We have thus constructed an experimental setup using Galinstan, a metal liquid at room temperature, to mimic the impactor core, and a mixture of water and industrial lubricant (Ucon Oil) with a tunable viscosity, to mimic the magma ocean. A given volume of Galinstan is initially placed at the top of a 1-m-deep tank inside a balloon, which is suddenly popped. The following dynamics are recorded using a high-speed camera. As shown in Fig. 1, changing the viscosity of the ambient fluid by a factor of 1000 does not change the mean falling speed significantly, which is in reasonable agreement with the inviscid scaling for the terminal velocity of the initial spherical mass of metal. Nevertheless,

*wacheul@irphe.univ-mrs.fr

Published by the American Physical Society under the terms of the Creative Commons Attribution 4.0 International license. Further distribution of this work must maintain attribution to the author(s) and the published article's title, journal citation, and DOI. 

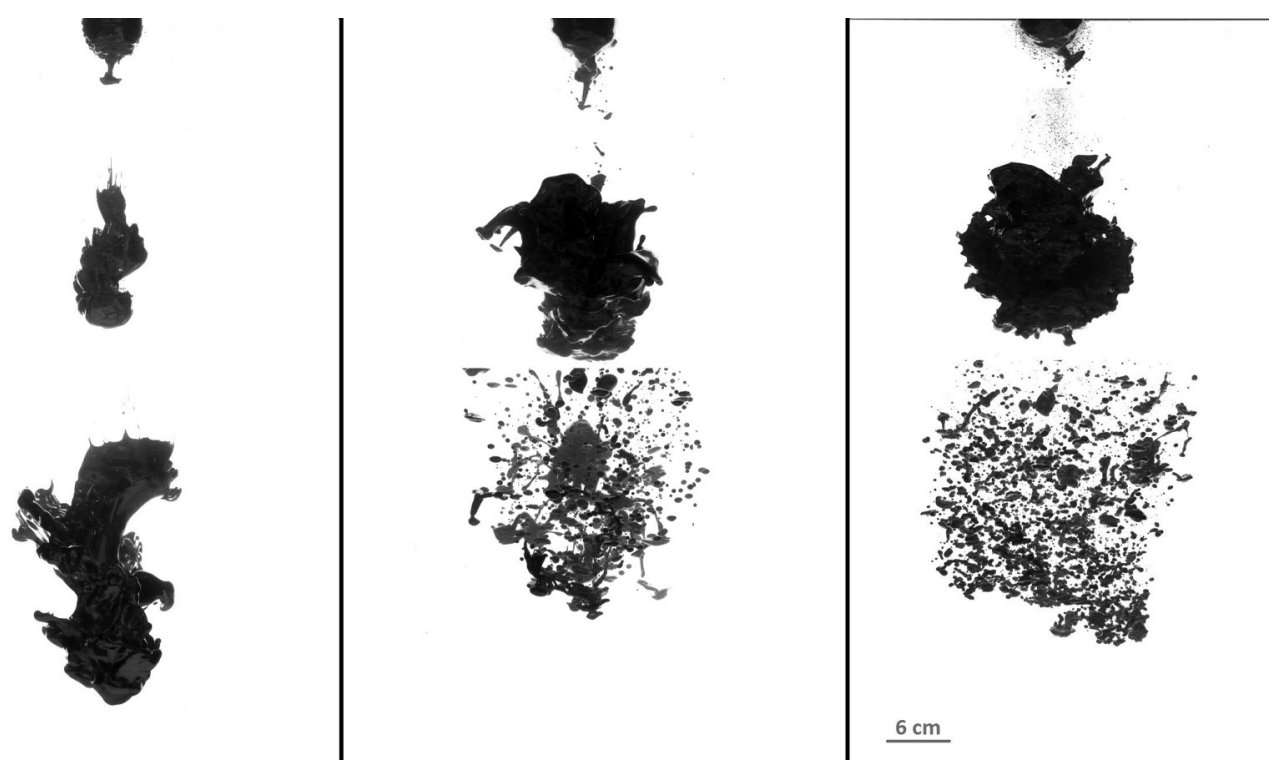

FIG. 1. Superimposition of three snapshots extracted at the same time (time interval of $0.26 \mathrm{~s}$ ) from three videos of the fall of a mass of liquid metal with an initial 31-mm spherical radius in an ambient fluid with different viscosities. From left to right the viscosity of the ambient fluid decreases by three orders of magnitude. The corresponding viscosity ratios between the two fluids are 700, 80, and 0.4 and the corresponding Reynolds numbers are 20, 175, and 35000 . The Weber number is 51 in all cases. See also the video at https://doi.org/10.1103/APS.DFD.2016.GFM.V0038

the viscosity ratio has a great influence on the fragmentation regime. At low viscosity ratio (Fig. 1, middle and right panels), the liquid metal is rolled in a turbulent vortex ring and stretched until the thin liquid metal sheet breaks up in a single event, in qualitative agreement with the fragmentation

(a)

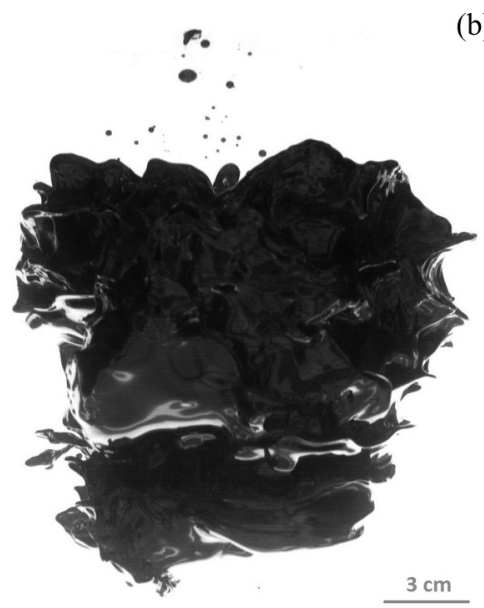

(b)

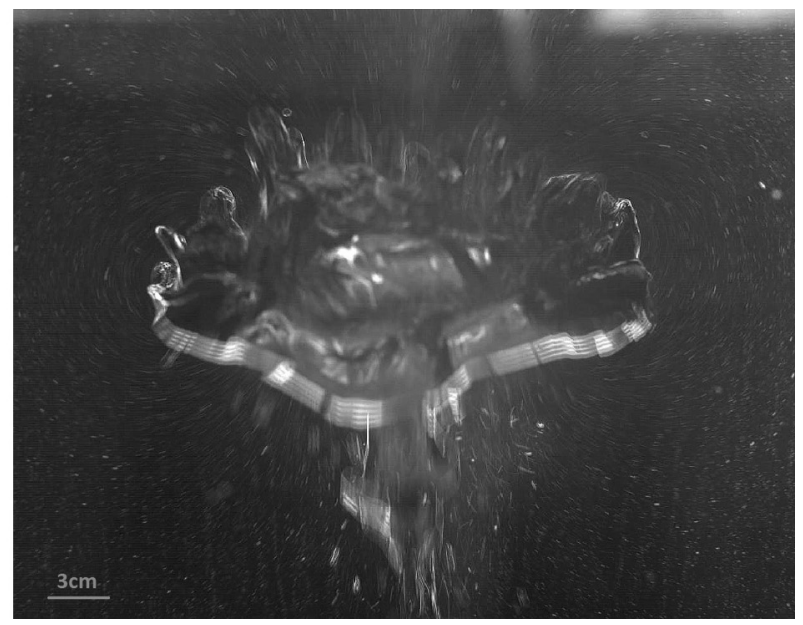

FIG. 2. Picture of the fall of a 31-mm-radius initial mass of liquid metal illuminated (a) from behind by a LED plane and (b) from the side by a ring light, with the ambient fluid previously seeded by a vertical plane of gas bubbles. The corresponding viscosity ratio is 8 , the Reynolds number is 1750 , and the Weber number is 51 . See also the video at https://doi.org/10.1103/APS.DFD.2016.GFM.V0038 


\section{FALL AND FRAGMENTATION OF LIQUID METAL IN A . . .}

mode found by Landeau et al. [6]. At the higher viscosity ratio (Fig. 1, left panel), vorticity tends to be localized in the liquid metal; it is not rolled up but stretched in long scales so that breakup rarely happens in a single event. Instead, the liquid metal is peeled away from the initial mass or separates from the rest via Rayleigh-Taylor instabilities.

When increasing the inertia of the flow, one could expect a dynamic similar to the immiscible case, corresponding to a turbulent thermal [8]. While the linear increase with depth of the mean radius of the metal blob agrees with the classical theory for turbulent thermals, this model systematically overestimates our measured velocities. We conjecture that this is due to the invalidity of the turbulent entrainment hypothesis, where the buoyant mass entrains fluid all along its surface with no significant initial momentum [9]. As shown in Fig. 2, we observe that the ambient fluid is engulfed at the rear of the liquid metal only, where strong stable vortices produce important velocity gradients in the ambient fluid. This flux produces an expansion of the liquid metal sheet that works against surface tension, producing an overpressure in the entrained liquid that could accelerate the falling velocity. The validation of this explanation now requires precise velocimetry in the ambient fluid.

The authors gratefully acknowledge financial support from the European Research Council under the European Union's Horizon 2020 research and innovation program (Grant Agreement No. 681835-FLUDYCO-ERC-2015-CoG).

[1] E. Kokubo and S. Ida, On runaway growth of planetesimals, Icarus 123, 180 (1996).

[2] T. Lebrun, H. Massol, E. Chassefière, A. Davaille, E. Marcq, P. Sarda, F. Leblanc, and G. Brandeis, Thermal evolution of an early magma ocean in interaction with the atmosphere, J. Geophys. Res. 118, 1155 (2013).

[3] R. Clift, J. R. Grace, and M. E. Weber, Bubbles, Drops, and Particles, 1st ed. (Academic, New York, 1978).

[4] D. Rubie, H. Melosh, J. Reid, C. Liebske, and K. Righter, Mechanisms of metal-silicate equilibration in the terrestrial magma ocean, Earth Planet. Sci. Lett. 205, 239 (2003).

[5] T. W. Dahl and D. J. Stevenson, Turbulent mixing of metal and silicate during planet accretion and interpretation of the Hf-W chronometer, Earth Planet. Sci. Lett. 295, 177 (2010).

[6] M. Landeau, R. Deguen, and P. Olson, Experiments on the fragmentation of a buoyant liquid volume in another liquid, J. Fluid Mech. 749, 478 (2014).

[7] R. Deguen, M. Landeau, and P. Olson, Turbulent metal-silicate mixing, fragmentation, and equilibration in magma oceans, Earth Planet. Sci. Lett. 391, 274 (2014).

[8] J. W. M. Bush, B. A. Thurber, and F. Blanchette, Particle clouds in homogeneous and stratifed environments, J. Fluid Mech. 489, 29 (2003).

[9] B. R. Morton, G. Taylor, and J. S. Turner, Turbulent gravitational convection from maintained and instantaneous sources, Proc. R. Soc. London Ser. A 234, 1 (1956). 